\title{
The functional variant of NTN1 contributes to the risk of nonsyndromic cleft lip with or without cleft palate
}

\author{
Dandan Li $\mathbb{B}^{1} \cdot$ Guirong Zhu' ${ }^{1} \cdot$ Shu Lou ${ }^{1} \cdot \operatorname{Lan~} \mathrm{Ma}^{1} \cdot$ Chi Zhang $^{1} \cdot$ Yongchu Pan ${ }^{1} \cdot$ Lin Wang $^{1}$
}

Received: 17 July 2019 / Revised: 22 October 2019 / Accepted: 29 October 2019 / Published online: 28 November 2019

(c) The Author(s) 2019. This article is published with open access

\begin{abstract}
Previous genome-wide association study of nonsyndromic cleft lip with or without cleft palate (NSCL/P) identified a susceptible variant (rs4791774). We hypothesized that the functional single nucleotide polymorphism (SNP) may be in linkage disequilibrium with this lead SNP. The potential functional SNP (rs4791331) was identified by bioinformatic analysis. A case-control study with 891 orofacial cleft cases and 830 controls was designed to investigate its association with orofacial cleft. The allele-specific DNA-protein binding preference was predicted by JASPAR database. Cell proliferation, cycle and apoptosis, luciferase activity and netrin-1 (NTNI) expression were examined after transfection with the rs4791331 C/T vector in HEK-293 and HEPM cell lines. Forty-six lip tissues of NSCL/P patients were collected to detect NTN1 expression. ntnla knockout zebrafish models were generated by CRISPR/Cas9 and observed with micro-CT. In the case-control study, the rs4791331-T allele was associated with an increased risk of nonsyndromic orofacial cleft $(\mathrm{OR}=$ $1.41,95 \% \mathrm{CI}=1.19-1.68)$, as well as the subgroups cleft lip only $(\mathrm{OR}=1.46,95 \% \mathrm{CI}=1.14-1.87)$ and cleft lip and palate $(\mathrm{OR}=1.58,95 \% \mathrm{CI}=1.27-1.96)$. The $\mathrm{T}$ allele of $\mathrm{rs} 4791331$ exhibited anti-apoptotic effects and promoted cell cycle progression at the G1/S transition. Decreased enhancer activity and reduced NTN1 expression following transfection of the T allele were observed. Carriers of the CT/TT genotypes showed significantly lower expression of NTNI than CC carriers. The $n t n 1 a^{-1-}$ zebrafish showed relatively wider intermaxillary fissures. These results indicate that rs4791331 $(\mathrm{C}>\mathrm{T})$ disrupted motif binding and led to abnormal expression of NTN1, which may be involved in the development of NSCL/P.
\end{abstract}

\section{Introduction}

Orofacial cleft is the most common congenital craniofacial malformation affecting humans [1], causing severe healthrelated problems as well as financial burdens for patients and their families [2]. Approximately $30 \%$ of orofacial clefts are syndromic and may be inherited in Mendelian fashion, whereas the remaining cases are nonsyndromic and appear to be induced by multiple genetic and environmental factors [3-6]. For nonsyndromic orofacial cleft (NSOC), the most

These authors contributed equally: Dandan Li, Guirong Zhu

Supplementary information The online version of this article (https:// doi.org/10.1038/s41431-019-0549-4) contains supplementary material, which is available to authorized users.

Yongchu Pan

Panyongchu@njmu.edu.cn

1 Jiangsu Key Laboratory of Oral Diseases, Nanjing Medical University, Nanjing 210029, China common types are nonsyndromic cleft lip with or without cleft palate (NSCL/P) and nonsyndromic cleft palate only.

Single nucleotide polymorphisms (SNPs) have attracted tremendous interest in recent years as potential susceptibility factors for complex traits. To date, dozens of genomic loci have been identified for NSOC by genome-wide association studies (GWASs) [7-14]. The data provided statistical support for the association between SNP markers and risk of NSOC.

However, the translation from statistical associations revealed by GWASs to the biological causes of NSOC is still a major challenge. The majority of these loci are located within intronic or intergenic regions. Understanding their function in gene regulation has been recognized as the key to connecting these phenotypes with DNA sequence variations. The most frequently affected elements are transcriptional enhancers and silencers, although the connection between sequence and function is currently poorly understood [15]. In most cases, SNPs within noncoding DNA are presumed to disrupt cisregulatory elements [16]. 
Previous studies showed that SNPs in strong linkage disequilibrium (LD) with the lead SNP could be functional for NSCL/P. As Liu et al. reported, three most-highly riskassociated SNPs at $1 \mathrm{p} 22$ were identified with alleledependent effect, affecting transcription factors binding ability or chromatin configuration [16]. Leslie et al. identified a common non-coding variant, rs227727, in $100 \%$ linkage disequilibrium with the most-strongly associated SNP identified in GWAS that alters the function of an enhancer [17]. Cvjetkovic et al. found a functional variant rs138557689 in intron of FZD6 by sequencing of the linkage region of $8 \mathrm{q} 21.3-24.12$, a candidate locus of NSCLP [18]. And, it was supposed to be an etiologic variant in linkage disequilibrium with rs41268753 for the etiology of cleft palate by Leslie et al. [19].

Our group previously performed the first GWAS for NSCL/P in a Chinese population, and rs4791774 (hg19 NC_000017.10:g.8932119 A > G, NC_000017.10:g.8932119 $\mathrm{A}>\mathrm{C}$ ), located at chromosome $17 \mathrm{p} 13.1$, showed a genomewide association with NSCL/P in this study [13] as well as in the targeted sequencing study of Asian case-parent trios [17]. This SNP is located in the intronic region near the transcription start site of netrin-1 (NTN1, Nucleotide RefSeq NM_004822.2 and exon numbering NC_000017.10). However, the functional mechanism underlying this association has not been scrutinized. Here, we first filtered the potentially functional SNP based on bioinformatic analysis and investigated its association with the risk of NSCL/P in a case-control cohort and then performed a series of experiments to explore its functional significance.

\section{Materials and methods}

\section{Bioinformatic analysis}

RegulomeDB and F-SNP for functional prediction were applied. HaploReg V4.1 software was used for LD analysis of rs4791774 to identify SNPs in high LD $\left(r^{2} \geq 0.8\right)$ with rs4791774 according to the LD information from the Asian population of 1000 Genomes Project Phase 1. The binding abilities of the transcription factors were predicted by JASPAR. The relative expression data of Ntnl in the 10.5-14.5-day mouse embryos (period of lip and palate fusion) were downloaded from Gene Expression Omnibus (GEO).

\section{Human subjects and DNA extraction}

This is an ongoing project for genetic study of NSOC, approved by the Ethics Review Committee (NJMUERC [2008] No. 20). The recruitment of study subjects was described preciously(Li et al. 2016). In brief, patients were recruited from three hospitals in Jiangsu Province between August 2008 and January 2015. Physical examination and medical records were obtained for clinical assessment. Patients with congenital isolated oral clefts without syndromic symptoms or other birth defects met the inclusion criteria of the case group. The controls were self-reported Chinese Han from the same region, and no known congenital anomalies were detected. Informed consent was provided by every volunteer of the study.

Approximately $2 \mathrm{ml}$ of venous blood was collected from each participant and stored in a tube containing ethylenediaminetetraacetic acid. Genomic DNA was extracted by the phenol-chloroform method. With the ABI Prism 7900HT system (Applied Biosystems), all samples were genotyped by a TaqMan allelic discrimination method with a call rate of $98.6 \%$. The sequences of the primers and probes are shown in Supplementary Table S1. Approximately $5 \%$ of the samples were randomly repeated, and the results were $100 \%$ concordant.

\section{Cell culture}

HEK-293 (ATCC-1573 ${ }^{\mathrm{TM}}$ ) and HEPM (human embryo palate mesenchyme, ATCC-1486 ${ }^{\mathrm{TM}}$ ) cell lines were purchased from the American Type Culture Collection (ATCC, Manassas, VA, USA) and cultured in Eagle's minimal essential medium with $10 \%$ fetal bovine serum (ATCC) and $1 \%$ Penicillin-Streptomycin Solution (Gibco, Foster City, CA, USA).

\section{Plasmid construction and transient transfection}

We cloned $\sim 700$ bp regions containing the individual alleles of rs4791331 (hg19 NC_000017.10:g.8932082 C > T). A plasmid containing the NTN1 fragment of the rs4791331 major $\mathrm{C}$ allele was inserted downstream of the luciferase gene in the PGL3-promoter vector (Promega, Madison, WI, USA) at the XbaI site. With this construct as the template, the minor allele was generated by the site-specific mutagenesis method. The nucleotide sequence of the recombinant plasmids was verified by DNA sequencing. (Supplementary Fig. S1). The PGL3-promoter vector with a random sequence inserted was used as the negative control.

Plasmids containing the $\mathrm{C}$ or $\mathrm{T}$ allele were transfected in cells using Lipofectamine 2000 (Invitrogen, Carlsbad, CA, USA).

\section{Cell proliferation, cycle and apoptosis analysis}

While cell culture continued for 24,48 , and $72 \mathrm{~h}$ after plasmid transfection, the Cell Counting Kit 8 (Dojindo, Kumamoto, Japan) was used to assess cell proliferation. The absorbance value at $450 \mathrm{~nm}$ was determined using a 
SpectraMAX 190 (Molecular Devices, USA). Absorbance at each time point was tested in triplicate with six independent repeats.

Forty-eight hours after transfection, cells were harvested and fixed overnight in $70 \%$ ice-cold ethanol followed by RNase A treatment and stained with $50 \mu \mathrm{g} / \mathrm{ml}$ of propidium iodide for DNA content analysis by flow cytometry on a FACSCalibur system (BD Medical Technology, USA). The results are expressed as a percentage of cells in each cell cycle phase. Cell apoptosis was detected by Annexin V-FITC/PI double staining $48 \mathrm{~h}$ after transfection.

\section{Dual-luciferase reporter assay}

The luciferase activity in the lysates was quantified with a dual-luciferase reporter assay system $48 \mathrm{~h}$ after transfection (Promega, Madison, WI). The luminescent reaction of the Renilla luciferase was simultaneously activated after the firefly luciferase reporter was measured as a stabilized luminescent signal. The firefly luciferase to Renilla luciferase ratio was considered the relative reporter activity. Independent triplicate experiments were performed for each plasmid construct.

\section{Lip tissue collection, RNA extraction, and quantitative real-time PCR}

Forty-six redundant lip tissue samples from NSCL/P patients who underwent surgery were collected. Total RNA was isolated using TRIzol reagent (Invitrogen, Carlsbad, CA, USA). The NTN1 mRNA levels relative to the transcription level of $G A P D H$ were quantified by quantitative reverse transcriptase-polymerase chain reaction (RT-qPCR) in an ABI 7900 HT instrument (Applied Biosystems, Foster City, CA, USA). All RT-qPCR reactions were performed using SYBR Green Real-Time PCR (TaKaRa, Shiga, Japan) according to the manufacturer's instructions. All reactions were performed in triplicate, and the relative gene expression was determined by the $2^{-\Delta \Delta \mathrm{Ct}}$ method [20]. The primers are listed in Supplementary Table S1.

\section{Zebrafish models}

Although vertebrates boast an enormous diversity of adult facial morphologies, the fundamental signaling pathways and cellular events that sculpt the nascent craniofacial structure in the embryo have proven to be highly conserved from zebrafish to man [21]. The zebrafish, affording experimental advantages toward investigating the normal function of genes associated with orofacial development, has served as a popular model since the 1990s. many researchers have employed this model to explore the functions of genes implicated in cleft lip and cleft palate $[16,22,23]$.

According to the synteny analysis (Supplementary Fig. S2) and phylogenetic tree analysis (Supplementary Fig. S3), the ntnla gene of zebrafish was identified as the orthologue of the human NTN1 gene with $86 \%$ similarity in an amino acid alignment. According to previous studies, ntnla expressed in the orofacial region during the facial development [24-26]. Therefore, we supposed that ntnla may affect the orofacial development of zebrafish without detecting its expression. CRISPR was designed to target a site in exon 1 of the ntnla gene. Mutagenesis and founder identification were carried out with the ntnla primers 5'-TTT CGC AAA AGT CCA GGT AGT G-3' (reverse) and 5'-TGG GTG TGT GTG ACT CCA TAT TG-3' (forward) (the recognition sequence and target sequence are listed in Supplementary Table S2). $2 \mu \mathrm{l}$ of sgRNA stock $(500 \mathrm{ng} / \mu \mathrm{l})$ was mixed with $2 \mu \mathrm{l}$ of recombinant Cas9 protein $(1 \mu \mathrm{g} / \mu \mathrm{l}$, PNA Bio, Thousand Oaks, CA) and incubated on ice for at least $10 \mathrm{~min}$ to allow formation of the sgRNA/Cas9 complex. $2 \mathrm{nl}$ of the injection mix was injected intracellularly in one-cell stage zebrafish embryos using glass needles and a micromanipulator. DNA was extracted from 10 pooled injected embryos and an uninjected control group at $48 \mathrm{~h}$ post fertilization using the HotShot protocol. Mutagenesis was determined by a T7 endonuclease assay. Positive clutches (F0 generation) were raised to adulthood and outcrossed against wild-type fish. Germline transmission was also determined by the $\mathrm{T} 7$ endonuclease assay. Positive clutches (F1 generation) were raised to adulthood and genotyped individually. Fish carrying the same variant were pooled as the founders of the heterozygous stable knockout line. The F1 generation was incrossed to obtain the homozygous ntn1a knockout F2 generation. Then, 3D images of the 4-month-old F2 generation were taken with a resolution of $18 \mu \mathrm{m}$ using SkyScan 1276 Micro-CT (Bruker, Germany).

\section{Statistical analysis}

Statistical analyses were performed using Version 9.1 of the $\mathrm{SAS}^{\bullet}$ software (SAS Institute Inc., Cary, NC, USA). For all graphs, data are shown as the mean \pm standard deviations (SD). An independent-sample $t$ test was applied for the comparison of different groups. The demographic characteristics of cases and controls were analyzed by Chisquare $\left(\chi^{2}\right)$ tests. Hardy-Weinberg equilibrium (HWE) of genotype frequencies in the control group was tested by Fisher's exact test. Odds ratios (ORs) and 95\% confidence intervals (CIs) for risk of NSOC were calculated using logistic regression analyses. All $P$ values were two-sided. The RNA-Seq count data with gene assignments downloaded from the GEO database were read and visualized by $\mathrm{R}$ (version 3.4.2) (R Core Team, 2017) and analyzed by one-way ANOVA. 
All the variant data has been submitted to ClinVar (Submission ID: SUB6440632, Organization ID: 6434903).

\section{Results}

\section{Identification of the potentially functional SNP by in silico analysis}

The score identifying regulatory elements by RegulomeDB and F-SNP was 4 (minimal binding evidence) and 0.194, respectively, for rs4791774 (Supplementary Table S3), indicating this index GWAS biomarker signal had yet unidentified causal variants. Instead of examining the lead SNP, one optional strategy is to analyze the functional SNPs in high LD with the lead SNP that modify the incidence of birth defects [27].

LD analysis showed that $\operatorname{rs} 4791331 \quad\left(r^{2}=0.99\right)$, rs9891446 $\left(r^{2}=0.8\right)$, and $\mathrm{rs} 36047638\left(r^{2}=0.8\right)$ were highly linked with rs4791774. Among them, rs4791331 was the functional site with the highest potential, with values of $2 \mathrm{~b}$ by RegulomeDB and 0.714 by F-SNP (Supplementary Table S3); the two software programs consistently predicted that rs4791331 was a transcription factor binding site.

\section{Association of rs4791331 and risk of NSCL/P}

A total of 856 unrelated NSOC cases and 823 healthy controls were recruited for this study. As shown in Table 1, the case group consisted of three subgroups: 266 cases of cleft lip only (CLO), 381 cases of cleft lip and palate (CLP), and 226 cases of cleft palate only (CPO). No statistical discrepancy in gender was identified between the cases and controls.

The distribution of three genotypes in the control group was consistent with HWE $(P=0.412)$. As presented in Table 2, the $\mathrm{T}$ allele was associated with an increased risk of

Table 1 Demographic characteristic information of the study samples

\begin{tabular}{|c|c|c|c|c|c|}
\hline & \multicolumn{2}{|c|}{ Cases $(N=873)$} & \multicolumn{2}{|c|}{$\begin{array}{l}\text { Controls } \\
(N=830)\end{array}$} & \multirow[t]{2}{*}{$P^{\mathrm{a}}$} \\
\hline & $N$ & $\%$ & $N$ & $\%$ & \\
\hline \multicolumn{6}{|l|}{ Gender } \\
\hline Male & 534 & 61.2 & 471 & 56.7 & 0.068 \\
\hline Female & 339 & 38.6 & 359 & 43.3 & \\
\hline \multicolumn{6}{|c|}{ Cases subtype } \\
\hline CLO & 266 & 30.5 & & & \\
\hline CLP & 381 & 43.6 & & & \\
\hline $\mathrm{CPO}$ & 226 & 25.9 & & & \\
\hline
\end{tabular}

$C L O$ cleft lip only, $C L P$ cleft lip with cleft palate, $C P O$ cleft palate only

${ }^{\mathrm{a}}$ Two-sided chi-square test
NSOC $\left(\mathrm{OR}=1.43,95 \% \mathrm{CI}=1.20-1.70, P=5.10 \times 10^{-5}\right.$, additive model). In the subtype analysis, the $\mathrm{T}$ allele was associated with CLO $(\mathrm{OR}=1.46,95 \% \mathrm{CI}=1.14-1.87, P=$ $0.003)$ and $\mathrm{CLP}(\mathrm{OR}=1.58,95 \% \mathrm{CI}=1.27-1.96, P=$ $5.50 \times 10^{-5}$ ) in the additive model. However, there was no statistical association between rs4791331 and CPO (OR = $1.21,95 \% \mathrm{CI}=0.93-1.59, P=0.159)$, confirming different genetic background among them.

\section{Effects of rs4791331 on cell apoptosis, cell proliferation, and the cell cycle}

As shown in Fig. 1a, the expression of the $\mathrm{T}$ allele led to a significant decrease in cell apoptosis, indicating that rs4791331-T exerts an antiapoptotic effect (HEK-293: $P=$ 0.011, HEPM: $P=0.007$ ). However, neither the rs $4791331 \mathrm{C}$ nor T allele had an effect on the proliferation of HEK-293 and HEPM cells (Supplementary Fig. S4). Cell cycle distribution showed that cell counts in the G1 phase were significantly decreased in HEK-293 $(P=0.001)$ and HEPM $(P=0.011)$ cells transfected with the vector with the rs791331 $\mathrm{T}$ allele compared with those with the $\mathrm{C}$ allele, accompanied by a substantial accumulation of the cells in S phase (HEK-293: $P=0.001$, HEPM: $P=0.013$ ) (Fig. $1 b, c$ ). Therefore, the rs4791331 T allele may have an antiapoptotic effect due to the enhancement of cell cycle progression at the G1/S transition in cells.

\section{Nuclear protein binds preferentially to the $\mathrm{T}$ allele sequence at the rs4791331 locus}

JASPAR revealed that rs4791331 mapped within the binding motifs of homeodomain transcription factors with SRY and TBP. A binding preference to the $\mathrm{T}$ allele over the $\mathrm{C}$ allele was predicted (Fig. 2a).

\section{rs4791331 C > T affects the expression of NTN1}

The luciferase reporter assay showed dramatic allelic differences in enhancer activity. The fragments with the $\mathrm{T}$ allele at rs4791331 showed significantly lower enhancer activity to drive luciferase gene expression in HEK-293 cells $(P<0.001)$ and HEPM cells $(P<0.001)$ than those with the $\mathrm{C}$ allele (Fig. 2b). Furthermore, compared with the $\mathrm{C}$ allele, the $\mathrm{T}$ allele was associated with lower expression of NTN1 (HEK-293: $P=0.049$, HEPM: $P=0.036$, Fig. 2c).

\section{NTN1 expression in human tissues and mouse craniofacial structures}

A total of 46 cleft lip tissues from NSCL/P patients were obtained and genotyped to assess the mRNA expression of 
Table 2 Association study of rs4791331 and NSOC susceptibility

\begin{tabular}{|c|c|c|c|c|c|c|c|c|}
\hline & \multicolumn{3}{|c|}{ Case } & \multicolumn{3}{|c|}{ Control } & \multicolumn{2}{|l|}{ Additive model } \\
\hline & $\mathrm{CC}$ & $\mathrm{CT}$ & TT & $\mathrm{CC}$ & $\mathrm{CT}$ & TT & OR $(95 \% \mathrm{CI})$ & $P$ \\
\hline NSOC & 531 & 283 & 42 & 585 & 214 & 24 & $1.43(1.20-1.70)$ & $5.10 \times 10^{-5}$ \\
\hline \multicolumn{9}{|c|}{ Subgroups } \\
\hline CLO & 160 & 91 & 11 & & & & $1.46(1.14-1.87)$ & 0.003 \\
\hline CLP & 224 & 129 & 21 & & & & $1.58(1.27-1.96)$ & $5.50 \times 10^{-5}$ \\
\hline $\mathrm{CPO}$ & 147 & 63 & 10 & & & & $1.21(0.93-1.59)$ & 0.159 \\
\hline
\end{tabular}

$C L O$ cleft lip only, $C L P$ cleft lip and palate, $C P O$ cleft palate only, $O R$ odds ratio, $95 \%$ CI 95\% confidence interval, additive model: rs4791331 CC/CT/TT
A

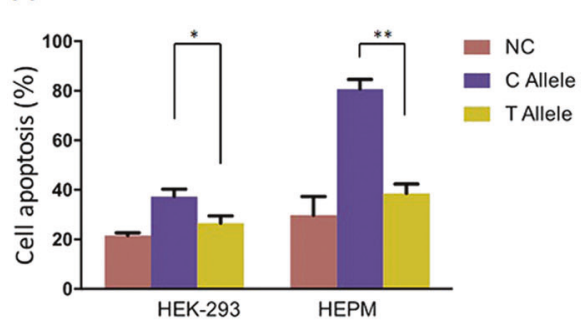

C

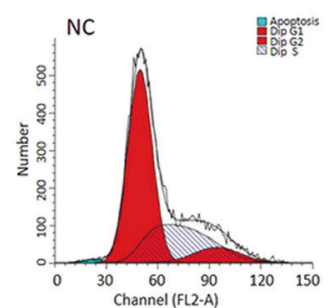

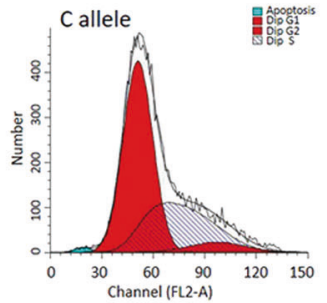
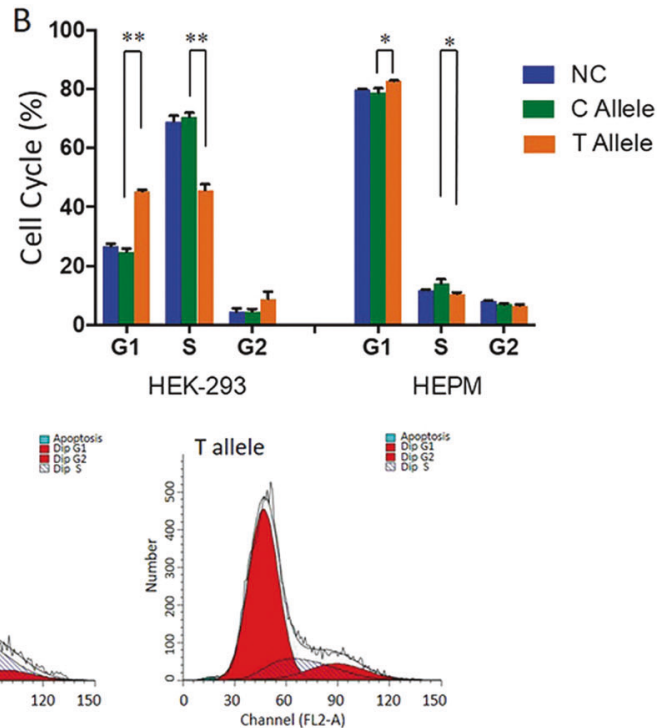

Fig. 1 a Effects of rs4791331 C/T allele on cell apoptosis in HEK-293 and HEPM cells. The apoptosis of cells was detected by Annexin VFITC/PI double staining method at $48 \mathrm{~h}$ after transfection with rs4791331-C, rs4791331-T, or nonsense control vector (NC). Results are shown as means \pm SD from three experiments, each with three replicates. b Representative histograms depicting cell cycle profiles of

cells transiently transfected with rs4791331-C/T allele or nonsense control vector, respectively. Cells were stained with PI and analyzed by flow cytometry. Various phases of the cell cycle in c HEK-293 and HEPM cells. The results are means of triplicate independent experiments. $(* P<0.05, * * P<0.01, * * * P<0.001$.)

NTN1 in vivo. The frequencies of the CC, CT, and TT genotypes of rs 4791331 were 26,18 , and 2, respectively, in the samples. We found that the CT and TT genotypes were significantly associated with decreased expression of NTN1 compared with the CC genotype $(P=0.014$, Fig. $2 d)$. In addition, NTN1 expression in the lymphocytes of the European population from the 1000 Genome database showed that individuals with the TT genotype had lower NTN1 expression than those with other genotypes $(P=0.047)$ (Fig. 2e).

Then, the relative expression of Ntn1 in the 10.5-14.5day mouse embryos (time period that lip and palate fusion occurred) was detected in the proximal maxillary process with an increasing trend from E10.5d to E13.5d and then a decrease on E14.5d. Ntn1 expression in the distal maxillary process increased from E11.5d to E12.5d and then leveled off (Supplementary Fig. S5).

\section{Zebrafish phenotype}

The ntnla knockout F2 generation of zebrafishes all survived. Under micro-CT, the $n t n 1 a^{-1-}$ mutant adult zebrafish at 4 months of age showed relatively wider intermaxillary fissures than the $n t n 1 a^{+/+}$zebrafish (Fig. 3, $P=0.057$ ). Although neither cleft lip nor cleft palate was observed, the wider intermaxillary fissure indicates the putative function of ntnla in orofacial fusion.

\section{Discussion}

GWASs have identified several SNPs related to NSCL/P in recent years. Because the majority of these loci are situated within intergenic or intronic regions, a mechanistic understanding of how they contribute to phenotypes is limited. 
A

a Sequence Risk Allele

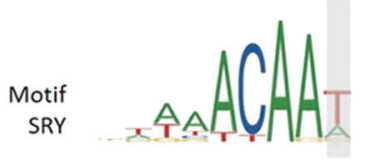

B

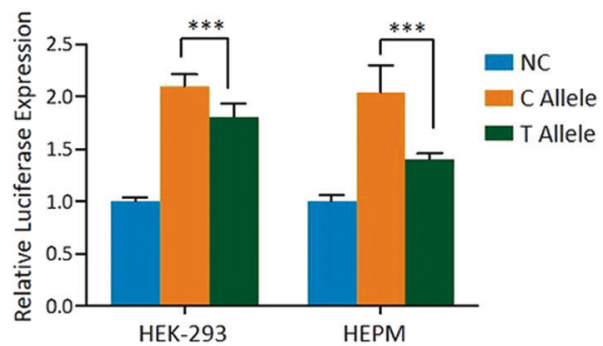

D

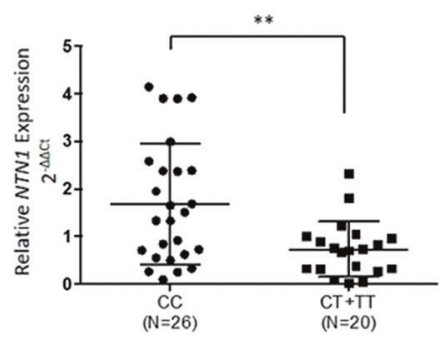

Fig. 2 a The position of rs4791331 in Chr17p13.1 reside within TBP (a) and SRY (b) DNA-binding motifs. b The plasmid constructed with the fragments of NTN1 containing the $\mathrm{C}$ or T allele of rs4791331 was transfected in HEK-293A cells and HEPM cells. A plasmid construct with a nonsense sequence was used as the negative control (NC). Binding ability assay of plasmids and transcription factors were detected. The firefly luciferase to Renilla luciferase ratio was considered the relative luciferase expression. c Quantitative real-time polymerase chain reaction analysis of NTN1 expression at $48 \mathrm{~h}$ after plasmids transfection in HEK-293 and HEPM cells. Independent

However, an increasing number of studies have demonstrated the important role of intronic mechanisms, such as altering RNA splicing activity [28], modulating RNA and protein expression [29] and changing the structure of proteins [30], which regulate the network for multifactorial diseases. A recent understanding of the control of gene expression has emerged as a key tool for connecting DNA sequence variation to phenotypes. A variant in the intronic region may alter the molecular mechanism of complex traits by affecting transcriptional activity through the altered binding ability of motifs. For instance, López Rodríguez et al. identified SNPs in a FOXA2-regulated transcriptional enhancer at a type 2 diabetes intronic locus that controls GCKR expression in liver cells [31]. For NSCL/P, Cvjetkovic et al. identified an SNP in intron 1 of FZD6 that creates an allele-specific protein-binding site and decreases

C
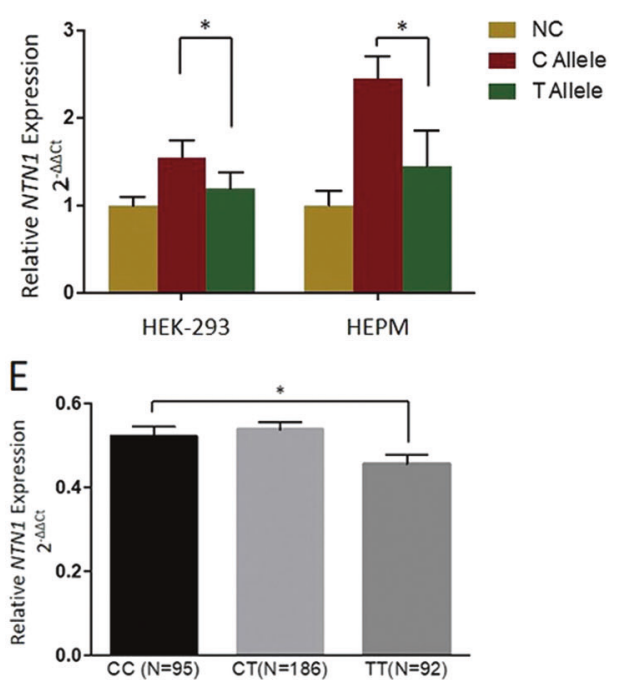

triplicate experiments were performed. Results are shown as mean values with the standard deviation (SD) normalized to GAPDH. d NTN1 expression levels in 46 NSCL/P cleft lip tissue samples. qRTPCR was used to test the NTN1 mRNA expression in 46 cleft lip tissues (26 samples of CC genotype, 18 samples with CT genotype and 2 of TT genotype). The results were normalized to $G A P D H$. Error bars indicate the standard deviations. e The e-QTL result of rs4791331NTN1 in the lymphocytes of European population from 1000 Genome database. $(* P<0.05, * * P<0.01, * * * P<0.001)$

promoter activity, which is involved in craniofacial development and contributes to NSCL/P by perturbing the WNT signaling pathway [18].

Before the GWAS of NSCL/P, the NTN1 gene in the chromosome $17 \mathrm{p} 13$ region was not associated with NSCL/P susceptibility. However, this region was shown to be related to NSCL/P risk by GWAS [11] and sequencing [17]. Notably, rs4791331 was once recognized as a "second hit" locus $(P=6.4100 \mathrm{E}-06)$ in Asian populations by Beaty et al. in a GWAS of case-parent trios of NSCL/P [7]. In this study, we hypothesized that rs4791331 in maximal LD with the lead SNP rs4791774 may be a functional SNP in this region according to the prediction of publicly available databases. In addition, the association study between rs4791331 and NSCL/P susceptibility confirmed that rs4791331 is associated 
A
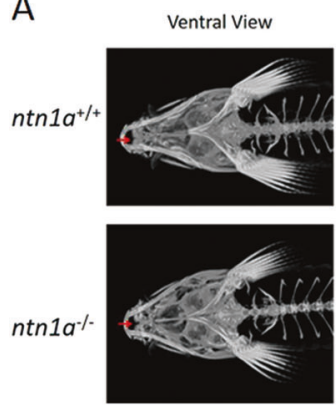
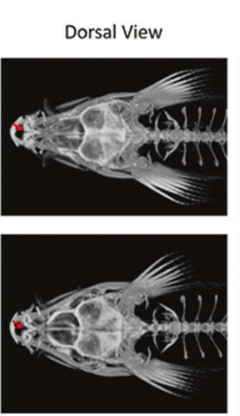

450 Profile View

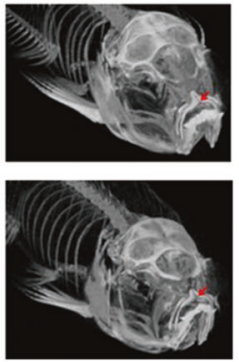

B

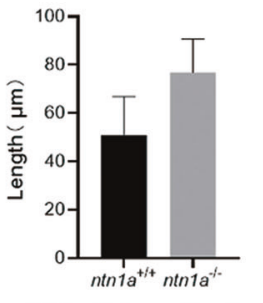

Fig. 3 a The ventral, dorsal and $45^{\circ}$ profile views of $\mathrm{F} 2$ generation zebrafish (4 months age) under Micro-CT (yellow array: mandibular width is the width between the inside border of the articulation; red array: intermaxillary fissure, the width were measured as the distance between the most anterior and inside point of the bilateral maxilla.

with the risk of NSCL/P, but no association between rs4791331 and CPO was found. Then, the mechanism was examined experimentally. According to the results of experiments in vitro, the mutant s4791331-T allele can downregulate the expression of NTN1 probably by altering the motif binding ability in HEK-293 and HEPM cells, followed by the reduction of cell apoptosis and modulation of cell cycles. It indicated that variant of rs4791331 may be related to the risk of NSCL/P and indicated the distinct pathogenic mechanisms between CLO, CLP, and CPO [32].

NTN1 is involved in various signaling pathways as a secreted protein that is expressed in the cell-extracellular matrix. For example, this protein is the effector molecule of IGF1/MEK/ERK and IGF1/ PI3K/AKT signaling pathways during cochlear hair cell protection [33] and modulates angiogenesis by the netrin-1/FAK/Src/CD151 signaling axis [34]. During embryonic development, NTN1 was detected in multiple tissues, such as the nervous system, vasculature, lung, pancreas, muscle, and mammary gland [35], and functioned as the major cue for midline attraction of the nervous system [36], regulating the progression of semicircular canal morphogenesis in the inner ear [37]. Moreover, the reduction of $\mathrm{Ntnl}$ expression in mouse embryos led to a lack of white spots of milk in the stomach and death during the perinatal period, consistent with an orofacial cleft phenotype [17, 38]. However, its role in the pathogenic mechanism of NSCL/P has never been addressed. In our study, the NTN1 gene variant was associated with modulation of cell apoptosis and the cell cycle. NTN1 was shown to regulate cell apoptosis in colorectal tumorigenesis [39] and modulate the cell cycle in angiogenesis [34], but the specific molecular mechanism remains unclear.

In conclusion, we found that the $\mathrm{C}>\mathrm{T}$ base change of rs4791331 resulted in abnormally low expression of NTN1, probably through disrupted motif binding, which may be involved in the development of NSCL/P. Our findings will help elucidate the etiology and genetic factors of NSCL/P. $n t n 1 a^{-/-}:$the homozygous knock out line, $n t n 1 a^{+/+}$: the wild homozygous line). b The comparison of the width of mandibular and the width of intermaxillary fissure between $n t n 1 a^{+/+}$and $n t n 1 a^{-l-}$ group. $(* * P<0.01)$

Acknowledgements The work was supported by the National Natural Science Foundation of China (81830031, 81570959), State Key Lab of Reproductive Medicine of Nanjing Medical University (JX116GSP20171416), the Priority Academic Program Development of Jiangsu Higher Education Institutions (PAPD-2018-87), the Natural Science Foundation of Jiangsu Province (BL2014073, BK20180667), the Natural Science Foundation of the Jiangsu Higher Education Institutions of China (15KJA320002, 18KJB320011), the Qing Lan Project (Yongchu Pan), Six Distinguished Talent (2016-WSW-008) and Jiangsu Provincial Medical Talent (ZDRCC2016023) and the Jiangsu Provincial Key Medical Discipline (zdxka2016026).

\section{Compliance with ethical standards}

Conflict of interest The authors declare that they have no conflict of interest.

Publisher's note Springer Nature remains neutral with regard to jurisdictional claims in published maps and institutional affiliations.

Open Access This article is licensed under a Creative Commons Attribution 4.0 International License, which permits use, sharing, adaptation, distribution and reproduction in any medium or format, as long as you give appropriate credit to the original author(s) and the source, provide a link to the Creative Commons license, and indicate if changes were made. The images or other third party material in this article are included in the article's Creative Commons license, unless indicated otherwise in a credit line to the material. If material is not included in the article's Creative Commons license and your intended use is not permitted by statutory regulation or exceeds the permitted use, you will need to obtain permission directly from the copyright holder. To view a copy of this license, visit http://creativecommons. org/licenses/by/4.0/.

\section{References}

1. Mangold E, Ludwig KU, Nothen MM. Breakthroughs in the genetics of orofacial clefting. Trends Mol Med. 2011;17:725-33.

2. Dixon MJ, Marazita ML, Beaty TH, Murray JC. Cleft lip and palate: understanding genetic and environmental influences. Nat Rev Genet. 2011;12:167-78.

3. Abid A, Maatouk F, Berrezouga L, Azodo C, Uti O, El-Shamy H, et al. Prevalence and severity of oral diseases in the Africa and Middle East region. Adv Dent Res. 2015;27:10-17. 
4. Christensen K, Fogh-Andersen P. Cleft lip (+/- cleft palate) in Danish twins, 1970-1990. Am J Med Genet. 1993;47:910-6.

5. Grosen D, Bille C, Pedersen JK, Skytthe A, Murray JC, Christensen K. Recurrence risk for offspring of twins discordant for oral cleft: a population-based cohort study of the Danish 1936-2004 cleft twin cohort. Am J Med Genet Part A. 2010;152A:2468-74.

6. Krauss RS, Hong M. Gene-environment interactions and the etiology of birth defects. Curr Top Dev Biol. 2016;116:569-80.

7. Beaty TH, Murray JC, Marazita ML, Munger RG, Ruczinski I, Hetmanski JB, et al. A genome-wide association study of cleft lip with and without cleft palate identifies risk variants near MAFB and ABCA4. Nat Genet. 2010;42:525-9.

8. Birnbaum S, Ludwig KU, Reutter H, Herms S, Steffens M, Rubini M, et al. Key susceptibility locus for nonsyndromic cleft lip with or without cleft palate on chromosome 8q24. Nat Genet. 2009;41:473-7.

9. Camargo M, Rivera D, Moreno L, Lidral AC, Harper U, Jones M, et al. GWAS reveals new recessive loci associated with nonsyndromic facial clefting. Eur J Med Genet. 2012;55:510-4.

10. Grant SF, Wang K, Zhang H, Glaberson W, Annaiah K, Kim CE, et al. A genome-wide association study identifies a locus for nonsyndromic cleft lip with or without cleft palate on $8 \mathrm{q} 24$. J Pediatr. 2009;155:909-13.

11. Leslie EJ, Carlson JC, Shaffer JR, Feingold E, Wehby G, Laurie CA et al. A multi-ethnic genome-wide association study identifies novel loci for nonsyndromic cleft lip with or without cleft palate on 2p24.2, 17q23 and 19q13. Human Mol. Genet. 2016;25:2862-2872.

12. Mangold E, Ludwig KU, Birnbaum S, Baluardo C, Ferrian M, Herms S, et al. Genome-wide association study identifies two susceptibility loci for nonsyndromic cleft lip with or without cleft palate. Nat Genet. 2010;42:24-26.

13. Sun Y, Huang Y, Yin A, Pan Y, Wang Y, Wang C, et al. Genomewide association study identifies a new susceptibility locus for cleft lip with or without a cleft palate. Nat Commun. 2015;6:6414.

14. Wolf ZT, Brand HA, Shaffer JR, Leslie EJ, Arzi B, Willet CE, et al. Genome-wide association studies in $\operatorname{dogs}$ and humans identify ADAMTS20 as a risk variant for cleft lip and palate. PLoS Genet. 2015;11:e1005059.

15. Edwards SL, Beesley J, French JD, Dunning AM. Beyond GWASs: illuminating the dark road from association to function. Am J Hum Genet. 2013;93:779-97.

16. Liu H, Leslie EJ, Carlson JC, Beaty TH, Marazita ML, Lidral AC, et al. Identification of common non-coding variants at $1 \mathrm{p} 22$ that are functional for non-syndromic orofacial clefting. Nat Commun. 2017;8:14759.

17. Leslie EJ, Taub MA, Liu H, Steinberg KM, Koboldt DC, Zhang Q, et al. Identification of functional variants for cleft lip with or without cleft palate in or near PAX7, FGFR2, and NOG by targeted sequencing of GWAS loci. Am J Hum Genet. 2015;96:397-411.

18. Cvjetkovic N, Maili L, Weymouth KS, Hashmi SS, Mulliken JB, Topczewski J, et al. Regulatory variant in FZD6 gene contributes to nonsyndromic cleft lip and palate in an African-American family. Mol Genet Genom Med. 2015;3:440-51.

19. Leslie EJ, Liu H, Carlson JC, Shaffer JR, Feingold E, Wehby G, et al. A genome-wide association study of nonsyndromic cleft palate identifies an etiologic missense variant in GRHL3. Am J Hum Genet. 2016;98:744-54.

20. Schmittgen TD, Livak KJ. Analyzing real-time PCR data by the comparative C(T) method. Nat Protoc. 2008;3:1101-8.

21. Mork L, Crump G. Zebrafish craniofacial development: a window into early patterning. Curr Top Dev Biol. 2015;115:235-69.

22. Duncan KM, Mukherjee K, Cornell RA, Liao EC. Zebrafish models of orofacial clefts. Dev Dyn Off Publ Am Assoc Anatomists. 2017;246:897-914.
23. Chiquet BT, Yuan Q, Swindell EC, Maili L, Plant R, Dyke J, et al. Knockdown of Crispld2 in zebrafish identifies a novel network for nonsyndromic cleft lip with or without cleft palate candidate genes. Eur J Hum Genet EJHG. 2018;26:1441-50.

24. Vanderlaan G, Tyurina OV, Karlstrom RO, Chandrasekhar A. Gli function is essential for motor neuron induction in zebrafish. Dev Biol. 2005;282:550-70.

25. Park KW, Urness LD, Senchuk MM, Colvin CJ, Wythe JD, Chien $\mathrm{CB}$, et al. Identification of new netrin family members in zebrafish: developmental expression of netrin 2 and netrin 4. Dev Dyn Off Publ Am Assoc Anatomists. 2005;234:726-31.

26. Opitz R, Hitz MP, Vandernoot I, Trubiroha A, Abu-Khudir R, Samuels M, et al. Functional zebrafish studies based on human genotyping point to netrin-1 as a link between aberrant cardiovascular development and thyroid dysgenesis. Endocrinology. 2015;156:377-88.

27. Dubois PC, Trynka G, Franke L, Hunt KA, Romanos J, Curtotti A, et al. Multiple common variants for celiac disease influencing immune gene expression. Nat Genet. 2010;42:295-302.

28. Lamech LT, Mallam AL, Lambowitz AM. Evolution of RNAprotein interactions: non-specific binding led to RNA splicing activity of fungal mitochondrial tyrosyl-tRNA synthetases. PLoS Biol. 2014;12:e1002028.

29. Yu Q, Shen XH, Li Y, Li RJ, Li J, Luo YY, et al. An intron mutation in the ACVRL1 may be associated with a transcriptional regulation defect in a Chinese family with hereditary hemorrhagic telangiectasia. PLoS ONE. 2013;8:e58031.

30. Akman HO, Kakhlon O, Coku J, Peverelli L, Rosenmann H, Rozenstein-Tsalkovich L, et al. Deep intronic GBE1 mutation in manifesting heterozygous patients with adult polyglucosan body disease. JAMA Neurol. 2015;72:441-5.

31. Lopez Rodriguez M, Kaminska D, Lappalainen K, Pihlajamaki J, Kaikkonen MU, Laakso M. Identification and characterization of a FOXA2-regulated transcriptional enhancer at a type 2 diabetes intronic locus that controls GCKR expression in liver cells. Genome Med. 2017;9:63.

32. Ludwig KU, Bohmer AC, Bowes J, Nikolic M, Ishorst N, Wyatt $\mathrm{N}$, et al. Imputation of orofacial clefting data identifies novel risk loci and sheds light on the genetic background of cleft lip +/cleft palate and cleft palate only. Hum Mol Genet. 2017; 26:829-42.

33. Yamahara K, Nakagawa T, Ito J, Kinoshita K, Omori K, Yamamoto N. Netrin 1 mediates protective effects exerted by insulinlike growth factor 1 on cochlear hair cells. Neuropharmacology. 2017;119:26-39.

34. Yang X, Li S, Zhong J, Zhang W, Hua X, Li B, et al. CD151 mediates netrin-1-induced angiogenesis through the Src-FAKPaxillin pathway. J Cell Mol Med. 2017;21:72-80.

35. Lai Wing Sun K, Correia JP, Kennedy TE. Netrins: versatile extracellular cues with diverse functions. Development. 2011;138: 2153-69.

36. Yung AR, Nishitani AM, Goodrich LV. Phenotypic analysis of mice completely lacking netrin 1. Development. 2015;142:3686-91.

37. Nishitani AM, Ohta S, Yung AR, Del Rio T, Gordon MI, Abraira VE, et al. Distinct functions for netrin 1 in chicken and murine semicircular canal morphogenesis. Development. 2017;144: 3349-60.

38. Bin JM, Han D, Lai Wing Sun K, Croteau LP, Dumontier E, Cloutier JF, et al. Complete loss of netrin-1 results in embryonic lethality and severe axon guidance defects without increased neural cell death. Cell Rep. 2015;12:1099-106.

39. Mazelin L, Bernet A, Bonod-Bidaud C, Pays L, Arnaud S, Gespach C, et al. Netrin-1 controls colorectal tumorigenesis by regulating apoptosis. Nature. 2004;431:80-84. 